\title{
Análisis fotogramétrico de las alteraciones cartilaginosas y de tejidos blandos nasales en pacientes intervenidos por labio-paladar hendido unilateral \\ Photogrammetric analysis of the alterations in nasal cartilaginous and soft tissue structures in patients operated for unilateral cleft lip and palate
}

\author{
Puente Espel, J.*, Ortiz Monasterio y Garay, F.**
}

Resumen

Los pacientes con fisura labio-palatina unilateral presentan alteraciones anatómicas de la nariz y de las estructuras odontológicas que afectan a la simetría facial, estética y al crecimiento. Estas alteraciones pueden corregirse durante la cirugía labial con corrección nasal primaria si se realiza una técnica quirúrgica correcta. En el caso de que la reparación primaria sea inadecuada, la región nasal mostrará deformidades como estigma del problema inicial.

El objetivo de este estudio es describir las alteraciones nasales y la relación que guardan las estructuras nasales con las unidades estéticas de la mejilla y del labio superior en 50 pacientes con labio y paladar hendido unilateral no sindrómico, operados mediante queiloplastia. Además, describimos los parámetros o mediciones nasales obtenidos en los pacientes con un resultado quirúrgico adecuado.

Las alteraciones anatómicas que con mayor frecuencia hemos encontrado en nuestra serie son: desplazamiento caudal de la cúpula alar en 48 pacientes $(96 \%)$; desviación del eje longitudinal nasal de 5 a $10^{\circ}$ en 24 pacientes (48\%); narina más grande del lado hendido en 28 pacientes (56\%); presencia de muesca en la narina en 18 pacientes (36\%); inserción de la base alar con el labio superior más caudal en el lado hendido en 35 pacientes $(70 \%)$ y desplazamiento medial de la unión del borde lateral del ala con la mejilla en el lado hendido en 34 pacientes $(68 \%)$.

Estos datos permiten suponer que es posible llevar a cabo una adecuada corrección en la primera intervención.
Palabras clave Labio-paladar hendido,

Deformidad nasal,

Rinoplastia primaria, Fotogrametría.

Nivel de evidencia científica III b
Patients with unilateral cleft lip and palate show anatomic disturbances related to nasal and odontologic tissues which directly affect facial symmetry, aesthetics and growth. These malformations can be repaired during the initial lip closure procedure with primary nasal correction, if a correct surgical technique is followed. If an incorrect surgical repair is performed, the nasal structures will show deformities which represent stigmata of the initial problem.

The aim of this study is to describe the nasal deformities and the relationship between the nasal structures and the cheek and superior lip aesthetic units in 50 patients with nonsyndromic unilateral cleft lip and palate that previously underwent a cheiloplasty. Furthermore, nasal measurements in patients with an adequate surgical result are also described.

The most frequent nasal anatomic abnormalities in our series were: caudal displacement of the alar dome in 48 patients $(96 \%)$; deviation of the nasal longitudinal axis in 5 to $10^{\circ}$ in 24 patients $(48 \%)$; larger nostril on the cleft side in 28 patients (56\%); nostril notch in 18 patients $(36 \%)$; alar base junction with the upper lip caudally oriented on the cleft side in 35 patients $(70 \%)$, and medial displacement of the tissue corresponding to the intersection of the alar lateral border with the cheek on the cleft side in 34 patients $(68 \%)$.

The data obtained show that an adequate surgical correction can be attained during the first intervention.
Key words Cleft lip and palate,

Cleft nose,

Primary rhinoplasty, Photogrammetry.

Level of evidence III b

* Cirujano Plástico. Servicio de Cirugía Plástica y Reconstructiva, Hospital General Dr. Manuel Gea González, México DF. México International Craniofacial Institute, Dallas, Texas, EE.UU.

** Profesor Emérito, Servicio de Cirugía Plástica y Reconstructiva, Hospital General Dr. Manuel Gea González y Universidad Nacional Autónoma de México, México DF, México. 


\section{Introducción}

Los pacientes con fisura labio-palatina unilateral presentan alteraciones anatómicas de la nariz y de las estructuras odontológicas que afectan a la simetría facial, a la estética y al crecimiento (1-4). Las anomalías nasales en este grupo de pacientes están ampliamente descritas en la literatura al respecto (1-5); producen deformidad nasal estética y pueden asociarse a problemas funcionales, generalmente asociados a desviación del septum nasal (6-7). Los dos aspectos más importantes a tener en cuenta en estas alteraciones anatómicas son: la asimetría de los cartílagos alares y la ausencia de volumen en el piso nasal.

La calidad de los resultados estéticos nasales en este tipo de pacientes depende de la técnica quirúrgica empleada y está condicionada por la experiencia del cirujano. Hasta hace pocos años, los cirujanos posponían la

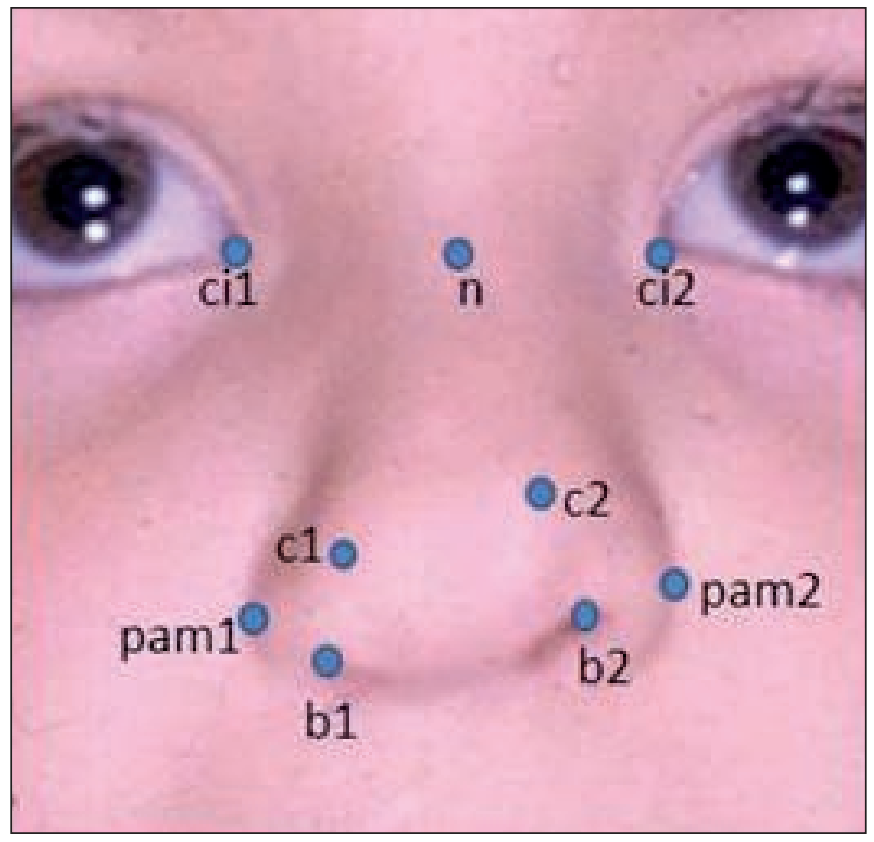

Fig. 1. A y B. Referencia antropométricas para la vista frontal.

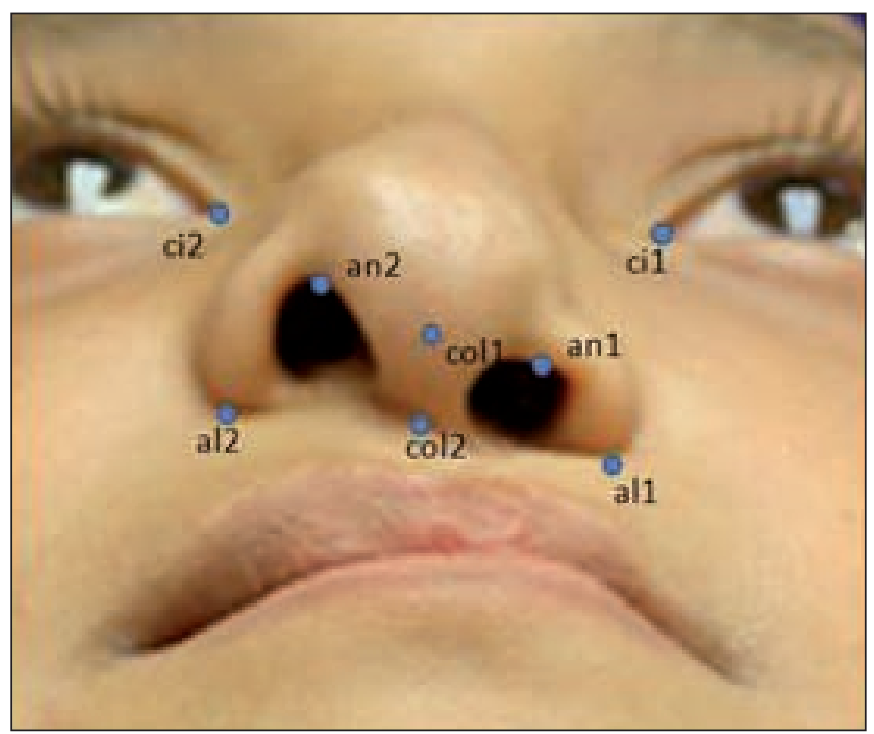

Fig. 2. A y B. Referencia antropométricas para la vista caudal. corrección nasal hasta que el paciente completaba el crecimiento facial pensando que la cirugía temprana limitaría el crecimiento de la nariz. A partir de los estudios de McComb, Anderl, Ortiz-Monasterio, Salyer y otros, se demostró que la cirugía nasal temprana, realizada con finura, no afecta al crecimiento de la nariz y que los cambios recogidos en publicaciones anteriores eran el resultado de cirugías agresivas, con presencia de tejido cicatricial y mutilación de elementos cutáneos y estructurales (5, 8-9). En la opinión del coautor de este artículo, las anomalías nasales pueden repararse en estos pacientes con éxito durante la queiloplastia y rinoplastia primaria. Es decir, las deformidades nasales que persisten como un estigma del problema inicial, pueden evitarse con una técnica quirúrgica correcta $(4-5,8)$.

Los objetivos del presente trabajo son, describir las alteraciones nasales y la relación que guardan las estructuras nasales con las unidades estéticas de la mejilla y del
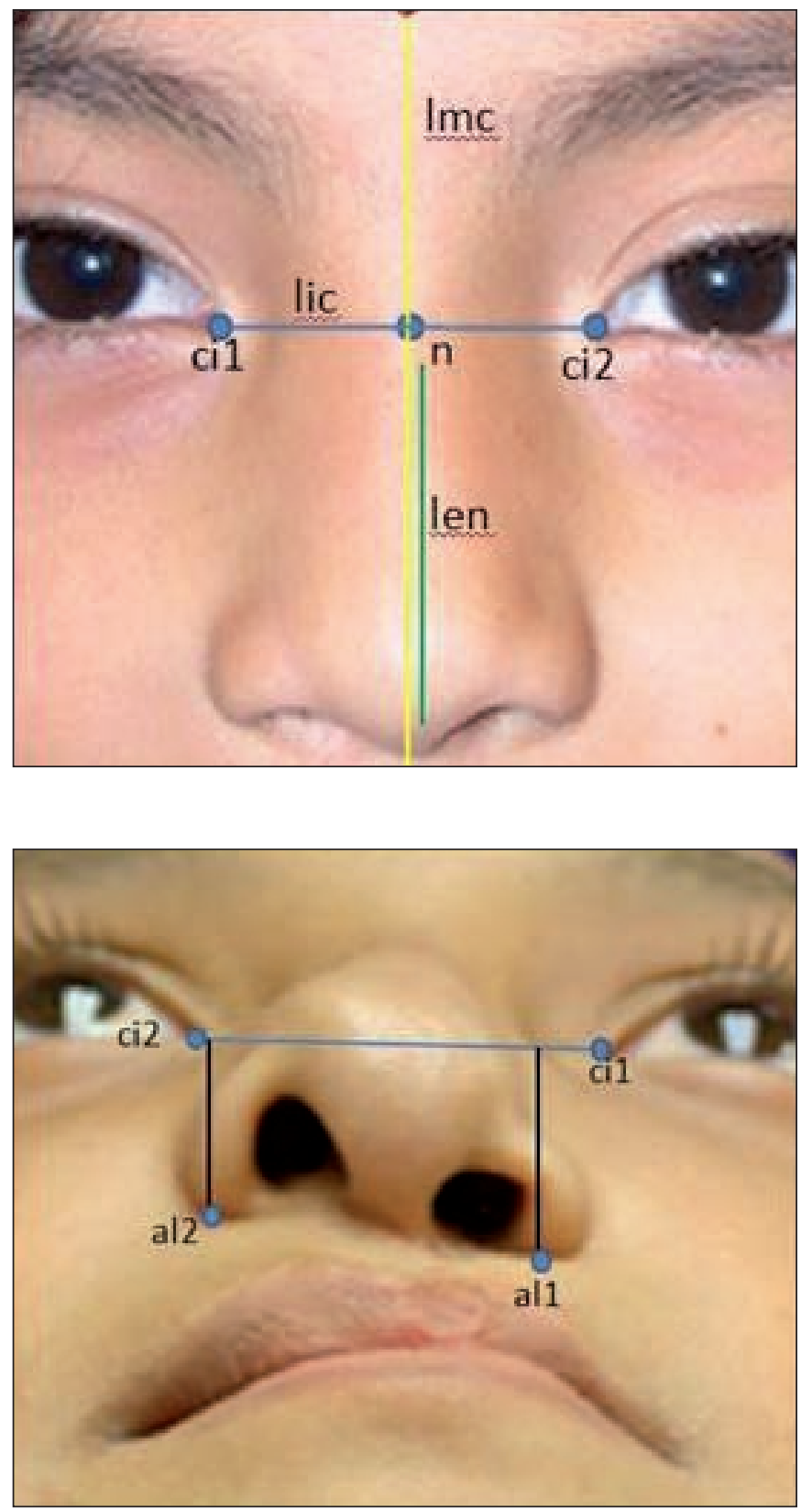
labio superior en pacientes con labio y paladar hendido unilateral operados de queiloplastia y, específicamente, describir los parámetros o mediciones nasales obtenidos en pacientes con un resultado quirúrgico adecuado.

\section{Material y método}

Seleccionamos de forma aleatoria 50 expedientes fotográficos y clínicos de pacientes con diagnóstico de labio y paladar hendido unilateral no sindrómico, de la base de datos del Hospital General Dr. Manuel Gea González (México DF, México), previamente operados de queiloplastia y rinoplastia primaria, que tuvieran fotografías pre y postoperatorias de la corrección nasal. Excluimos a aquellos pacientes con labio y paladar hendido unilateral en los que ya se hubiera realizado una rinoplastia secundaria y eliminamos los expedientes fotográficos o clínicos incompletos.

Empleamos fotografías digitales estandarizadas (1200 puntos por pulgada) con vistas frontal y caudal, realizadas en el postoperatorio a la edad en la que el paciente fue incluido en el estudio (intervalo de 4 a 14 años).
El análisis consistió en puntos, ángulos y relaciones de líneas antropométricas. Localizamos 10 puntos antropométricos en las fotografías, e implementamos 6 líneas y sus ángulos. Las referencias antropométricas utilizadas se muestran en las figuras $1 \mathrm{~A}-2 \mathrm{~A}, 1 \mathrm{~B}$ y $2 \mathrm{~B}$; las definiciones conceptuales están descritas en la Tabla 1 y las variables para los análisis de las vistas frontal y caudal se presentan en la Tabla 2.

\section{Análisis estadístico}

Para las variables sociodemográficas realizamos estadística descriptiva en la que se recogieron variables categóricas mediante frecuencias y porcentajes, y variables numéricas mediante medidas de tendencia central y dispersión (media, mediana o moda y desviaciones estándar). A las evaluaciones de los 3 expertos se les realizó prueba de concordancia mediante el estadístico Kappa y el coeficiente de correlación intraclase. Analizamos las variables mediante el software SPSS versión 16,0 y todos los datos fueron capturados en una hoja de recolección de datos diseñada en Excel (Office 2010).

Tabla I. Referencias antropométricas para la vista frontal

\begin{tabular}{|l|l|l|}
\hline \multicolumn{1}{|c|}{ Abreviatura } & \multicolumn{1}{c|}{ Nombre } & Definición \\
\hline Lic & Línea intercantal & Línea que une ambos cantos mediales de la fisura palpebral \\
\hline Lmc & Línea media facial & Linea media sagital facial \\
\hline Len & Eje nasal & $\begin{array}{l}\text { Trazo de la línea intercantal [n] al punto de mayor proyección de } \\
\text { la punta nasal [no] }\end{array}$ \\
\hline ci & Canto medial & Punto más medial de la fisura palpebral \\
\hline apn & Punto del ápice narinal & Punto más craneal de la narina \\
\hline pam & Punto de unión del ala nasal-mejilla & Punto más lateral del reborde alar \\
\hline ca & Punto de la cúpula alar & Punto más prominente de la cúpula alar \\
\hline $\mathrm{n}$ & Punto intercantal & Punto medio de distancia entre los cantos mediales \\
\hline pn & Punta nasal & Punto de mayor proyección de la punta nasal \\
\hline
\end{tabular}

Referencias antropométricas para la vista caudal

\begin{tabular}{|l|l|l|}
\hline \multicolumn{1}{|c|}{ Abreviatura } & \multicolumn{1}{c|}{ Nombre } & Definición \\
\hline Lic & Línea intercantal & Línea que une ambos cantos mediales de la fisura palpebral \\
\hline lec & Línea del eje mayor columelar & Trazo del punto medio columelar 1 al punto medio columelar 2 \\
\hline lmc & Línea media facial & Línea media sagital facial \\
\hline bn & Punto de la base narinal & Punto más caudal de la narina \\
\hline apn & Punto del ápice narinal & Punto más craneal de la narina \\
\hline col1 & Punto medio columelar 1 & Punto medio columelar en su ápice \\
\hline col2 & Punto medio columelar 2 & Punto medio columelar en su base \\
\hline
\end{tabular}


Tabla II. Análisis para la vistas frontal y caudal

Análisis de la vista frontal

\begin{tabular}{|l|l|}
\hline \multicolumn{1}{|c|}{ Nombre } & \multicolumn{1}{c|}{ Definición } \\
\hline Posición de la cúpula alar & $\begin{array}{l}\text { Distancia vertical de la línea intercantal [lic] al punto más prominente de la cúpula alar [ca], paralelo a } \\
\text { la línea media facial [lmc] }\end{array}$ \\
\hline Posición del ápice narinal & $\begin{array}{l}\text { Distancia vertical de la línea intercantal [lic] al punto más craneal de la narina [apn], paralelo a la línea } \\
\text { media facial [lmc] }\end{array}$ \\
\hline Ángulo de desviación nasal & Ángulo entre el eje nasal [len] y la línea media facial [lmc] \\
\hline Posición alar & Distancia horizontal del punto de unión ala nasal-mejilla a la línea media facial [lmc] \\
\hline
\end{tabular}

Análisis de la vista caudal

\begin{tabular}{|l|l|}
\hline \multicolumn{1}{|c|}{ Nombre } & Definición \\
\hline Ángulo del eje mayor columelar & Ángulo entre el eje columelar [lec] y la línea media facial [lmc] \\
\hline Índice del eje mayor narinal & Índice generado entre la distancia del eje mayor narinal lado hendido / lado no hendido \\
\hline Simetría del eje mayor narinal & Ángulo entre el eje mayor narinal [lec] y la línea media facial [lmc] \\
\hline Muesca narinal & Presencia o ausencia de muesca narinal \\
\hline $\begin{array}{l}\text { Unión de la base del ala nasal con } \\
\text { el labio superior }\end{array}$ & $\begin{array}{l}\text { Distancia vertical de la línea intercantal [lic] al punto de la base narinal [bn], paralelo a la línea media } \\
\text { facial [lmc] }\end{array}$ \\
\hline
\end{tabular}

Tabla III. Resultados de la fotogrametría para la vista frontal

\begin{tabular}{|c|c|c|c|c|}
\hline Variable & $\begin{array}{l}\text { Tamaño de la muestra } \\
\qquad(\mathrm{n}=\mathbf{5 0})\end{array}$ & $\begin{array}{l}\text { Número de estudios } \\
\text { de la muestra con la } \\
\text { característica }\end{array}$ & $\begin{array}{c}\text { Porcentaje } \\
\text { de la muestra }\end{array}$ & $\begin{array}{l}\text { Medidas de } \\
\text { estadística } \\
\text { descriptiva }\end{array}$ \\
\hline Desplazamiento craneal de la cúpula alar & 1 & $1 / 50$ & 2 & $\begin{array}{l}\text { Desplazamiento del } \\
3 \%\end{array}$ \\
\hline $\begin{array}{l}\text { Cúpula alar sin desplazamiento } \\
\text { en el eje vertical }\end{array}$ & 1 & $1 / 50$ & 2 & \\
\hline Desplazamiento caudal de la cúpula alar & 48 & $48 / 50$ & 96 & $\begin{array}{l}\text { Rango de desplaza- } \\
\text { miento: } 1,4-34,2 \% \\
\quad(\text { media } 9,62 \%)\end{array}$ \\
\hline $\begin{array}{l}\text { Desviación del eje longitudinal nasal } \\
\text { Sin desviación }\left(0^{\circ}\right) \\
\leq 2^{\circ} \\
2.1-5^{\circ} \\
5-10^{\circ} \\
\geq 10.1^{\circ}\end{array}$ & $\begin{array}{c}1 \\
4 \\
5 \\
24 \\
16\end{array}$ & $\begin{array}{c}1 / 50 \\
4 / 50 \\
5 / 50 \\
24 / 5 \\
16 / 50\end{array}$ & $\begin{array}{c}2 \\
8 \\
10 \\
48 \\
32\end{array}$ & $\begin{array}{l}\text { Rango de desviación: } \\
0-13^{\circ}\left(\text { media } 7,8^{\circ}\right)\end{array}$ \\
\hline $\begin{array}{l}\text { Desplazamiento medial de la unión del } \\
\text { borde lateral del ala con la mejilla en el } \\
\text { lado hendido }\end{array}$ & 34 & $34 / 50$ & 68 & $\begin{array}{c}\text { Rango: } \\
0,7-41,1 \% \\
\text { (media } 11,2 \text { ) }\end{array}$ \\
\hline $\begin{array}{l}\text { Desplazamiento lateral de la unión del } \\
\text { borde lateral del ala con la mejilla en el } \\
\text { lado hendido }\end{array}$ & 14 & $14 / 50$ & 28 & $\begin{array}{c}\text { Rango: } \\
0,6-25 \% \\
\text { (media } 6,8)\end{array}$ \\
\hline $\begin{array}{l}\text { Unión base alar con mejilla sin desplaza- } \\
\text { miento lateral }\end{array}$ & 2 & $2 / 50$ & 4 & \\
\hline
\end{tabular}

\section{RESULTADOS}

Presentamos los resultados de las mediciones para la vista frontal y caudal en la Tablas III y IV, respectivamente. Además, realizamos una evaluación de todos los estudios fotográficos por parte de 3 expertos, quienes ca- lificaron los resultados quirúrgicos obtenidos como adecuados o inadecuados.

Comparamos los resultados de las mediciones con la apreciación de los expertos. Con base en la evaluación realizada de manera independiente por los 3 expertos de 
Tabla IV. Resultados de la fotogrametría para la vista basal

\begin{tabular}{|l|c|c|c|c|}
\hline \multicolumn{1}{|c|}{ Variable } & $\begin{array}{c}\text { Tamaño de la muestra } \\
(\mathbf{n = 5 0 )}\end{array}$ & $\begin{array}{c}\text { Número de estudios } \\
\text { de la muestra con la } \\
\text { característica }\end{array}$ & $\begin{array}{c}\text { Porcentaje } \\
\text { de la muestra }\end{array}$ & $\begin{array}{c}\text { Medidas de } \\
\text { estadística } \\
\text { descriptiva }\end{array}$ \\
\hline Narina más pequeña del lado hendido & 19 & $19 / 50$ & 38 & $\begin{array}{c}\text { Rango: } \\
1,1-17,3 \% \\
\text { (media 14,6) }\end{array}$ \\
\hline Narina más grande del lado hendido & 28 & $28 / 50$ & 56 & $\begin{array}{c}\text { Rango: } \\
\text { (me-66,6\% } \\
\text { (media 17,3) }\end{array}$ \\
\hline Dimensión igual de las narinas & 3 & $3 / 50$ & 6 & \\
\hline Presencia de muesca & 18 & $18 / 50$ & 36 & \\
\hline Simetría narinal & 23 & $23 / 50$ & 46 & $\begin{array}{c}\text { Rango: } \\
\text { (media 3,3) }\end{array}$ \\
\hline $\begin{array}{l}\text { Inserción de la base alar con el labio } \\
\text { superior más craneal en el lado hendido }\end{array}$ & 15 & $15 / 50$ & 30 & $\begin{array}{c}\text { Rango: } \\
\text { (media 6,98) }\end{array}$ \\
\hline $\begin{array}{l}\text { Inserción de la base alar con el labio } \\
\text { superior más caudal en el lado hendido }\end{array}$ & 35 & $35 / 50$ & 70 & \\
\hline
\end{tabular}

los 50 archivos fotográficos de la muestra de pacientes estudiados, 13 pacientes cumplieron con datos de adecuada técnica quirúrgica y 37 pacientes con un resultado quirúrgico inadecuado, con una consistencia interna de 0,74 y una correlación de $0,42-0,51(\mathrm{p}=0,005)$.

\section{Discusión}

Una técnica quirúrgica deficiente en la cirugía del labiopaladar hendido unilateral se asocia a una mala colocación de la cúpula alar. En el presente estudio encontramos que en el 97,3\% de los pacientes con una cúpula alar mal colocada, ésta se encuentra en posición más caudal en relación al lado no hendido. También observamos una variación del 3,4 al 34,2 \% en este desplazamiento. Además, cabe citar que esta variable determina en gran medida la definición de los puntos luminosos en la punta nasal.

Una disección cuidadosa y completa, así como la manipulación adecuada de los cartílagos alares durante la disección subcutánea con tijera curva, determinan la disposición del orificio narinal. Nuestro estudio demostró que un 56\% (28 pacientes) presentó una narina más grande del lado hendido (con un rango en puntos porcentuales en relación a la narina del lado no hendido del 0,8 al $66,6 \%$ ). Un $38 \%$ (19 pacientes) presentó una narina del lado hendido más pequeña en relación con la narina del lado no hendido (rango del 1,7 al 27,5\%). En 3 pacientes (6\%) encontramos una dimensión del eje mayor narinal igual en el lado hendido en relación con el no hendido.

Además, la presencia de una muesca en el reborde narinal habla directamente de la disección incompleta de los cartílagos alares a nivel del reborde narinal superior. La muesca se encontró en el 36\% (18 pacientes) de todos los pacientes.

La manipulación cuidadosa de los tejidos durante la disección cartilaginosa determina la simetría del orificio narinal del lado hendido en relación al lado no hendido. Llama la atención que el $92 \%$ de los casos de pacientes del grupo correspondiente a un resultado adecuado (12 de 13 pacientes) mostró una simetría narinal en contra del $29,7 \%$ en el grupo de un resultado inadecuado (11 de 36 pacientes).

En esta investigación encontramos también que el 68\% de los pacientes (34 casos) presentó una base alar más medial del lado hendido en relación al lado no hendido. Un 28\% (14 pacientes) presentó una disposición más lateral y un $4 \%$ ( 2 pacientes) mostró simetría en ambas longitudes.

Otros estudios antropométricos como los de Farkas (10), presentan dificultades técnicas por la complejidad de sus mediciones y la falta de reproductibilidad de resultados en ausencia de estudios imagenológicos complejos (11-14). Esta investigación destaca el uso de herramientas fotogramétricas y clínicas sencillas para el análisis de la simetría en pacientes con labio y paladar hendido unilateral.

¿Por qué es importante la simetría? Se acepta que la simetría de la cara y, específicamente la de la nariz, considerada en sus diferentes segmentos, es una característica de normalidad y de belleza $(8-9,15-18)$. Una simetría menor resulta aceptable al observador; no obstante, una asimetría considerable o mayor, mantiene el estigma de deformidad de la patología presente.

La obtención de simetría se logra con una técnica quirúrgica óptima (8), en la cual cada una de las estructuras anatómicas se coloque en la misma situación que la del lado sano. Los aspectos morfológicos que determinarán una disposición simétrica de las estructuras nasales incluyen: 
- En la región de la punta nasal, una adecuada posición de las estructuras cartilaginosas, lograda mediante la disección precisa y completa de los cartílagos alares y la correcta liberación de los cartílagos alares de los cartílagos laterales superiores.

- En la región de la base nasal, una adecuada disección y colocación de los tejidos tanto en el plano sagital, que confiere la misma altura a la base nasal con respecto al lado no hendido, como en la unión mejilla-ala nasal (plano transverso), que proporciona la misma amplitud con respecto al lado no hendido.

- En el orificio narinal, una completa y correcta liberación de los cartílagos alares que provee la forma redondeada (con ausencia de muesca) y un tamaño semejante a la narina del lado sano.

Las alteraciones nasales residuales que representan un estigma de la presencia de labio y paladar hendido unilateral son: la falta de proyección y de definición de la punta nasal, la asimetría de narinas, la presencia de muesca en la narina y la asimetría de la base nasal del lado hendido en los planos sagital y transverso.

Es nuestra firme convicción que la ausencia de estas alteraciones en los pacientes operados por labio-paladar hendido unilateral, está directamente relacionada con una técnica quirúrgica adecuada y refinada, por lo que es muy importante tener en cuenta todos estos detalles anatómicos en la planificación cuidadosa de la cirugía para poder evitarlas.

\section{Conclusiones}

Las alteraciones anatómicas más frecuentes encontradas en esta serie de pacientes sometidos a cirugía por labio-paladar hendido unilateral fueron:

- Desplazamiento caudal de la cúpula alar.

- Desviación del eje longitudinal nasal de $5^{\circ}$ a $10^{\circ}$.

- Narina más grande del lado hendido.

- Presencia de muesca narinal.

- Inserción de la base alar con el labio superior más caudal en el lado hendido.

- Desplazamiento medial de la unión del borde lateral del ala con la mejilla en el lado hendido.

La cirugía inicial de la fisura labial requiere un conocimiento preciso de las estructuras de la nariz. Una técnica quirúrgica adecuadamente realizada conlleva una posición de los tejidos cartilaginosos y blandos muy semejante a la del lado no hendido, lo que nos permite suponer que es posible lograr una corrección adecuada en la primera intervención quirúrgica.

\section{Dirección del autor}

Dr. Jordi Puente Espel

International Craniofacial Institute

11970 N. Central Expressway, Suite 270

Dallas, Texas, USA, 75243

e-mail: jordiespel@hotmail.com
Bibliografía

1. Hopper RA, Cutting C, Grayson B. Cleft lip and palate. En: Thorne CH, Beasley RW, Aston SJ et al. Grabb and Smith's Plastic Surgery. 6 a edición. Philadelphia, PA: Ed Wolters Kluwer/Lippincott Williams \& Wilkins, 2007. Pp: 201-225.

2. Van Waes HJM, Stockli PW. Atlas de odontología pediátrica. 2a edición. Barcelona: Ed Masson, 2002. p: 350

3. Van Beek AL, Hatfield AS, Schnepf E. Cleft rhinoplasty. Plast Reconstr Surg 2004; 114(4): 57e-69e.

4. Guyuron B.: Late cleft lip nasal deformity. Plast Reconstr Surg 2008; 121(4):1-11.

5. Salyer KE. Primary correction of the unilateral cleft lip nose: a 15-year experience. Plast Reconstr Surg 1986; 77(4): 558-566.

6. Farkas LG, Lindsay WK. Morphology of the adult face following repair of unilateral cleft lip and palate in childhood. Plast Reconstr Surg 1973; 52(6): 652-655.

7. Byrd HS, Salomon J. Primary correction of the unilateral cleft nasal deformity. Plast Reconstr Surg 2000; 106(6): 1276-1286.

8. Ortiz Monasterio F, Ruas EJ. Cleft lip rhinoplasty: The role of bone and cartilage grafts. Clin Plast Surg 1989; 16: 177-186.

9. Velázquez JM, Ortiz-Monasterio F. Primary simultaneous correction of the lip and nose in the unilateral cleft lip. Plast Reconstr Surg 1974; 54(5): 558-563.

10. Lindsay WK, Farkas LG. The use of anthropometry in assessing the cleft-lip nose. Plast Reconstr Surg 1972; 49(3): 286-293.

11. Huempfner-Hierl H, Hemprich A, Hierl T. Results of a prospective anthropometric and functional study about aesthetics and nasal respiration after secondary rhinoplasty in cleft lip and palate patients. J Craniofac Surg 2009; 20: 1863-1875.

12. Ferrario VF, Sforza C, Tartaglia GM, Sozzi D, Caru A. Three-dimensional lip morphometry in adults operated on for cleft lip and palate. Plast Reconstr Surg 2003; 111(7): 2149-2156.

13. Kim SK, Cha BH, Lee KC, Park JM. Primary correction of unilateral cleft lip nasal deformity in Asian patients: Anthropometric evaluation. Plast Reconstr Surg 2004; 114(6): 1373-1381.

14. Suzuki H, Yamaguchi T, Furukawa M. Rhinologic computed tomographic evaluation in patients with cleft lip and palate. Arch Otolaryngol 1999; 125(9): 10001004.

15. Cronin TD, Denkler KA. Correction of the unilateral cleft lip nose. Plast Reconstr Surg 1988; 82(3): 419-432.

16. Ortiz-Monasterio F, Serrano Rebeil A, Valderrama M, Cruz R. Cephalometric measurements on adult patients with nonoperated cleft palates. Plast Reconstr Surg Bull 1959; 24(1): 53-61.

17. Millard DR. Earlier correction of the unilateral cleft lip nose. Plast Reconstr Surg 1982; 70(1): 64-73.

18. Trigos MI, Guzman ME. Analisis de la incidencia, prevalencia y atencion del labio y paladar hendido en Mexico. Cir Plast 2003; 13(1): 35-39. 